\title{
Variabilidad del viento, nivel del mar y temperatura en la costa norte de Chile*
}

\author{
OSCAR PIZARRO A., SAMUEL HORMAZABAL F., \\ ANTONIO GONZALEZ C. y ELEUTERIO YAÑEZ R. \\ Escuela de Ciencias del Mar \\ Universidad Católica de Valparaíso \\ Casilla 1020, Valparaíso, Chile
}

\begin{abstract}
RESUMEN. En este trabajo se describe el comportamiento del viento, la temperatura y nivel del mar en Arica $\left(18^{\circ} 28^{\prime} \mathrm{S}\right)$,

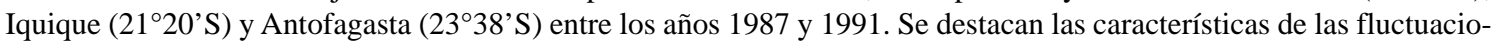
nes interdiarias y las relaciones entre las diferentes variables. Los resultados muestran que el viento del suroeste predomina en la zona costera durante todo el año. Este presenta un ciclo anual caracterizado por valores máximos en verano y mínimos en invierno. La dirección principal sobre la cual ocurren las perturbaciones del viento coincide con la dirección de la línea de costa. Las variaciones de baja frecuencia (menores a $0,2 \mathrm{cpd}$ ) del nivel del mar presentan alta coherencia espacial y se comportan como ondas no dispersivas que viajan de norte a sur con una velocidad de $300 \mathrm{~cm} / \mathrm{s}$. La temperatura del mar en Arica y Antofagasta no muestran buena relación con el viento. En Iquique las perturbaciones de la temperatura y el viento con períodos mayores a cinco días se encuentran bien relacionadas. El tiempo de respuesta de la temperatura a las variaciones del viento fue entre uno y dos días. El nivel del mar, en general, no mostró buena relación con el viento, siendo su variabilidad dominada principalmente por ondas atrapadas a la costa.
\end{abstract}

Palabras claves: Ondas atrapadas, nivel del mar, vientos, temperatura, surgencia, norte de Chile.

\section{Coastal wind, sea level and temperature variability in the north of Chile}

\begin{abstract}
This paper describes wind behaviour, temperature and sea level in Arica $\left(18^{\circ} 28^{\prime} \mathrm{S}\right)$, Iquique (21 $\left.{ }^{\circ} 20^{\prime} \mathrm{S}\right)$, and Antofagasta (233'ㅇ) between 1987 and 1991. Relationships between these variables and the features of their fluctuations are emphasized. Results show that southwest winds prevail all year round. Nevertheless, an annual cycle is present with characteristic summer maxima and winter minima. The main direction over which wind disturbances occur is coincident with the coastline direction. Low frequency variations ofthe sea level (less than $0,2 \mathrm{cpd}$ ) show high spatial coherence and behave like non dispersive waves travelling from north to south at $300 \mathrm{~cm} / \mathrm{sec}$. Sea temperature in Iquique relates to the wind, specially in disturbances with over five day periods. Response time of temperature to a wind variation was near one-two días. The sea level, in general, did not show a good relationship with the wind, its variability is dominated by coastal trapped waves.
\end{abstract}

Key words: Coastal trapped waves, sea level, wind, temperature, upwelling, northern Chile.

\section{INTRODUCCION}

Por varias décadas se ha registrado el viento en diferentes aeropuertos comerciales cercanos a la costa

* Trabajo financiado por Proyecto FONDECYT N 0481/92. a lo largo del país. Esta información, junto a registros de nivel y temperatura del mar medidos al interior de las principales bahías, conforman largas series de tiempo que pueden ser empleadas en el estudio de la variabilidad del océano costero. Diversos 
trabajos han utilizado estas observaciones de viento para estimar el transporte Ekman en superficie y de este modo, obtener información sobre la intensidad y variabilidad temporal de la surgencia en algún punto de la costa de Chile (Arcos y Navarro, 1986; Fuenzalida, 1990).

La surgencia se caracteriza por el ascenso de aguas frías y por el descenso del nivel del mar junto a la costa en respuesta al transporte superficial originado por el viento. De este modo, además de la información de viento, resulta interesante complementar los estudios de surgencia con registros costeros de temperatura y nivel del mar. Existen diversos fenómenos relevantes en la dinámica costera y oceánica en general, que pueden ser abordados mediante el uso de esta información. Las características de las series de tiempo actualmente disponibles, permiten estudiar fenómenos de escala interdiaria, como los trabajos mencionados anteriormente, hasta fenómenos que involucran variaciones interanuales como El Niño Oscilación del Sur (Enfield y Allen, 1980; Fonseca, 1987).

El objetivo del presente trabajo es explorar, desde un punto de vista estadístico, el comportamiento temporal del viento favorable a la surgencia, la temperatura del mar y el nivel del mar en la costa norte de Chile, con énfasis en las fluctuaciones comprendidas entre dos días y algunas semanas. El análisis se orientó hacia la descripción de estas variaciones y cómo ellas se relacionan entre sí, con el fin de conocer algunos aspectos de la variabilidad temporal del sistema de surgencia en la región.

\section{ANTECEDENTES}

La circulación atmosférica superficial a gran escala de la región centro y norte de Chile, es dominada por la presencia del anticiclón subtropical del Pacífico Sur Oriental (APSO). SU centro se localiza entre los $25^{\circ}$ y $30^{\circ} \mathrm{S}$ y $l o s ~ 90^{\circ}$ y $105^{\circ} \mathrm{W}$ y su influencia típicamente alcanza los $38^{\circ} \mathrm{S}$, aún cuando durante el verano austral éste puede penetrar hacia el sur sobrepasando los $45^{\circ} \mathrm{S}$ (Fuenzalida, 1971). La circulación atmosférica asociada al APSO tiene una importante componente geostrófica, la cual es modificada por fricción en las cercanías de la superficie. En la zona norte de Chile, ésta da lugar a un viento predominantemente del sur y suroeste, cuya componente a lo lar- go de la costa es responsable de la surgencia costera.

Además de la componente de gran escala asociada al APSO, existe un forzamiento térmico asociado a la diferencia de temperatura entre la atmósfera sobre las aguas y la atmósfera sobre el desierto. Las diferencias de presión relacionadas con este contraste térmico, junto con la rotación terrestre pueden generar un viento a lo largo de la costa que refuerza la componente favorable a la surgencia del viento de gran escala, resultando en un sistema de retroalimentación positiva (Enfield, 1981b). Este forzamiento es afectado por las fluctuaciones anuales de la radiación en la superficie del mar y de la tierra, incidiendo directamente sobre el ciclo anual de los vientos. Otro factor que interviene a una escala de espacio regional-local y de tiempo interdiaria es la acción de depresiones costeras forzadas sin ópticamente que se propagan por la capa límite atmosférica, produciendo variaciones en los vientos superficiales (Rutllant, 1986).

En la región, juegan un rol preponderante las variaciones interanuales producidas por el fenómeno El Niño Oscilación del Sur (ENOS). Asociados a este fenómeno se han observado importantes anomalías positivas en la temperatura del mar, en la temperatura del aire y en el nivel del mar. También se han reconocido efectos de este fenómeno sobre los vientos regionales (Enfield, 1981a; Romero y Garrido, 1985; Montecinos, 1991); sin embargo, en este caso, no se ha logrado establecer un patrón de comportamiento característico para los diferentes eventos.

Las anomalías mensuales del nivel del mar y de la temperatura del mar relacionadas al ENOS presentan una notable coherencia espacial a lo largo de la costa oeste de todo el continente americano. Estas anomalías son generadas en el Pacífico Ecuatorial Central y viajan por el ecuador hacia la costa de Sudamérica como ondas de baja frecuencia (MacCreary, 1976). Posteriormente continúan su propagación en dirección a los polos como ondas atrapadas a la costa (Enfield y Allen, 1980).

La variabilidad del nivel del mar y, en general, diferentes aspectos de la dinámica costera, a escala interdiaria ha sido ampliamente estudiada en las costasde Perú (Smith, 1978; Brink et al., 1983; Romea y Smith, 1983; Cornejo-Rodríguez y Enfield, 1987; Huyer et al., 1987, 1991). De acuerdo a estos trabajos una gran parte de la variabilidad observada en la costa noroccidental de Sudamérica sería explicada 
por ondas atrapadas a la costa originadas en la zona ecuatorial. Estas oscilaciones producen perturbaciones en las velocidades, en los campos de presión y densidad, afectando de manera importante la surgencia costera (Huyer et al., 1987). En la costa norte de Chile, Pizarro (1991) encontró perturbaciones que se propagan de norte a sur con velocidades cercanas a los $250 \mathrm{~km} /$ día, la cual concuerda con lo esperado para ondas no forzadas atrapadas a la costa. Estas observaciones permiten suponer que parte de la energía de origen ecuatorial alcanza las costas de Chile afectando el ambiente físico donde se desarrollan fenómenos de connotación local, como es el caso de la surgencia costera. Para períodos El Niño, la energía de origen ecuatorial que alcanza nuestras costas puede ser de uno o más órdenes de magnitud mayor que la energía en los períodos no El Niño y sus efectos en los registros costeros han sido bien identificados (Fonseca,1987).

\section{MATERIALES Y METODOS}

Los datos de viento y presión atmosférica utilizados en este trabajo son valores diarios obtenidos a partir de observaciones de 10 minutos cada seis horas ( 02 , 08,14 y $20 \mathrm{hrs}$ en horario local) registradas en estaciones meteorológicas ubicadas en los aeropuertos comerciales de Chacalluta (Arica), Diego Aracena (Iquique) y Cerro Moreno (Antofagasta), entre enero de 1987 y diciembre de 1991. Para el caso de la presión atmosférica, el valor diario empleado fue un promedio simple de las cuatro observaciones, mientras que para el viento se utilizó un promedio de los registros de las 14 y $20 \mathrm{hrs}$. Estas horas corresponden al período de mayor intensidad del viento dentro del ciclo diario. Se eligió este promedio ya que el viento de las 14 y 20 horas mostró gran persistencia en dirección y magnitud. Por el contrario, en las mediciones de las 02 y 08 horas el viento es débil y presenta gran variabilidad en la dirección, lo cual disminuye considerablemente la confiabilidad de las observaciones, especialmente cuando éstas son registros puntuales. Este procedimiento involucra una sobre-estimación del viento promedio diario; sin embargo, la disminución del viento durante la madrugada es producto de una fluctuación diurna que se restringe a una región cercana a la costa (Beardsley et al., 1987). De esta forma, el promedio de las mediciones obtenidas durante la tarde, además de ser confiables, interpretaría de mejor manera el régimen

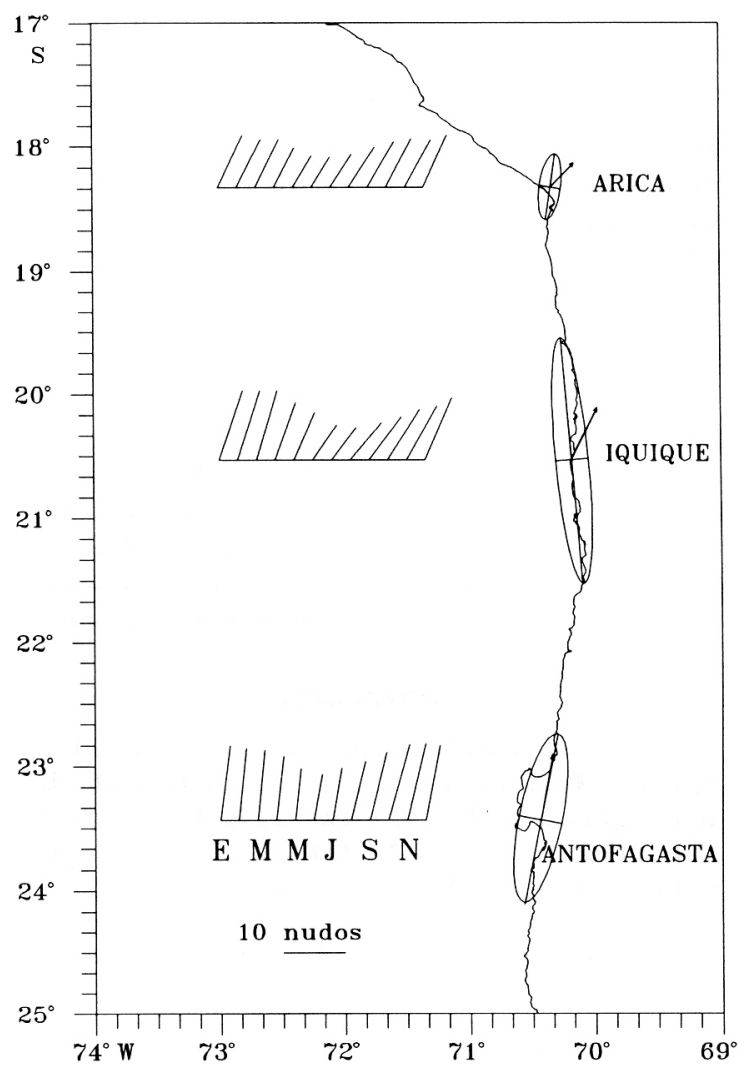

Figura 1. Ubicación geográfica de las estaciones. Vectores promedios mensuales del viento en Arica, Iquique y Antofagasta (1987-91) y elipses asociadas a los ejes de máxima y mínima varianza. La flecha en el centro de la elipse representa el viento promedio del período.

de vientos que actúa sobre el océano.

Los datos de nivel del mar corresponden a promedios diarios de alturas horarias de la marea y comprenden un período similar al indicado para las variables meteorológicas. Estos datos fueron corregidos para eliminar las fluctuaciones debido a los cambios en la presión atmosférica. Este procedimiento es conocido como corrección por efecto de barómetro invertido y consiste en sumar un centímetro al nivel del mar por cada milibar de presión sobre un valor base, comúnmente $1000 \mathrm{mb}$. Esta variable se denomina nivel del mar ajustado (NMA). La información original de nivel del mar fue obtenida de los registros efectuados en estaciones mareo gráficas dependientes del Servicio Hidrográfico y Oceanográfico de la Armada que se encuentran ubicadas en las bahías de Arica, Iquique y Antofagasta (Fig. 1). La tem- 

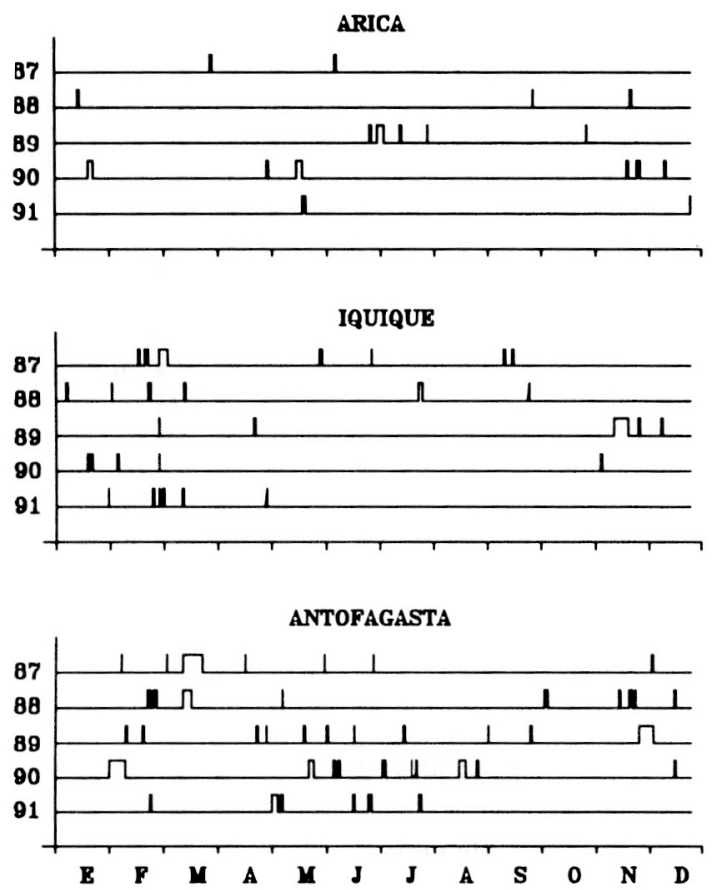

Figura 2. Series del nivel del mar empleadas en el trabajo. Los saltos en la línea indican las brechas presentes en las series.

peratura del mar en la costa (TMC) se registró en el mismo lugar y corresponde a una observación diaria, tomada manualmente mediante un termómetro oceanográfico durante la mañana. Al igual que las otras variables la TMC analizada comprende el período entre enero de 1987 y diciembre de 1991.

Todas las series originales fueron inicialmente graficadas para remover la información errónea y para detectar posibles discontinuidades en las series. En general la información meteorológica no presentó brechas ni datos visiblemente incorrectos. La cantidad de datos faltantes en estas series fue muy reducida y ninguna brecha superó los dos días. En este caso, los valores fueron estimados mediante interpolación lineal simple. Para los vientos la interpolación se efectuó en base a las componentes norte-sur y este-oeste. A diferencia de las variables meteorológicas, las series de nivel del mar presenta- ron brechas importantes (Fig. 2). Para completar aquellas mayores a 4 días, se utilizó un procedimiento orientado a mantener las características espectrales de las series. En primer lugar se calcularon los coeficientes de Fourier a ambos lados de la brecha, tomando un segmento de largo igual a ésta. Posteriormente se empleó el promedio de los coeficientes obtenido de los datos a ambos lados de la brecha para construir una serie sintética que fue sumada a la recta que unía ambos extremos.

En relación a la temperatura del mar, no se dispuso de información para los meses de enero, febrero y marzo del año 1987 en Iquique. En Antofagasta se excluyó del análisis un período comprendido entre julio y septiembre de 1989 (Fig. 11), debido a un conjunto de datos evidentemente erróneos. En general, salvo lo ya mencionado, estas series no presentaron brechas mayores a dos días, constituyendo una cifra inferior al 2\% de los datos. En estas brechas los valores fueron estimados mediante interpolación lineal.

Para el análisis espectral se empleó el método descrito por Bendat y Piersol (1972) usando la transformada rápida de Fourier. Las series fueron divididas en un número variable de trozos de igual longitud y se calculó el poder espectral de cada uno de ellos. Previamente, a cada segmento se le eliminó la tendencia lineal y se le aplicó una ventana hanning. Posteriormente las estimaciones espectrales fueron promediadas para cada frecuencia, obteniéndose una estimación de mayor significado físico y estadísticamente más confiable. Los grados de libertad de las estimaciones finales corresponden al doble del número de trozos empleados. Ocasionalmente para obtener mayor confianza se promediaron estimaciones dentro de un rango de frecuencias. El intervalo de confianza y la resolución en ancho de banda, asociados a estas estimaciones, se muestra al interior de las diferentes figuras. En el análisis de correlación se utilizaron diferentes trozos cuyo largo se especifica en cada ocasión. Estos fueron previamente corregidos para eliminar la tendencia lineal con el fin de obtener una relación representativa de las fluctuaciones que se quería analizar, evitando la contaminación producida por variaciones de muy baja frecuencia. Los límites de confianza para establecer si la correlación estimada es significativamente diferente de cero, fueron calculados usando un número de grados de libertad de acuerdo a la escala de tiempo integral propuesta por Davies (1976) y Sciremamano (1979). 


\section{RESULTADOS}

\section{El viento}

La Figura 1 muestra los vectores promedios del viento, los ejes de máxima y mínima varianza de cada localidad y el ciclo anual calculado en base a los promedios mensuales del período 1987-91. La media en Iquique y Arica es hacia el noreste, mientras que en Antofagasta es aproximadamente hacia el norte. En las tres localidades el viento muestra una importante componente favorable a la surgencia, la cual prevalece durante todo el año. Las elipses asociadas a la varianza de los vientos son angostas y se alinean notablemente con la dirección de la costa. Más del $80 \%$ de la varíanza observada en los tres lugares se encuentra asociada a esta dirección.

El ciclo anual presentó características similares en las tres localidades, con valores máximos durante el período cálido (entre noviembre y marzo) y mínimos en invierno. A diferencia de Arica e Iquique el viento en Antofagasta fue predominantemente paralelo a la costa, con un vector promedio sobre el eje de máxima varianza.

La variabilidad de baja frecuencia fué analizada mediante valores promedios mensuales de las componentes norte-sur y este-oeste. Esta variabilidad puede ser separada en una componente estacional y otra no-estacional. La segunda, obtenida como la diferencia entre las series y el ciclo anual, es relativamente pequeña, representando el 18,10 y $12 \%$ de la varianza total de la componente norte-sur en Arica, Iquique y Antofagasta respectivamente. En la Figura 3 se muestran estas componentes para las tres loca- lidades. Visualmente es posible notar que estas fluctuaciones se encuentran muy poco relacionadas entre sí y no presentan una variación a una escala de tiempo interanual.

La Figura 4 muestra las componentes perpendicular y a lo largo de la costa de las observaciones diarias en Arica, Iquique y Antofagasta. Durante el período cálido existe un viento continuo favorable a la surgencia con una varianza relativamente pequeña. En este período casi no se observan eventos de vientos hacia el sur o períodos de relajamiento. Durante los meses de invierno la variabilidad interdiaria es mayor, aún cuando el viento predominante sigue siendo desde el suroeste. En estos meses es posible observar cortos períodos de relajamiento o de vientos hacia el sur, especialmente en Iquique y Antofagasta.

De las tres localidades, Arica presentó vientos de menor intensidad, con una variabilidad también menor. En Antofagasta y Arica los vientos alcanzan magnitudes máximas a partir de fines de septiembre, en cambio en Iquique se presentan los vientos máximos entre diciembre y marzo. Este desfase puede ser apreciado en las series diarias (Fig. 4) y en los vectores promedios mensuales (Fig. 1).

La Figura 5 muestra los espectros de energía, coherencia y fase de la componente del viento a lo largo de la costa. La energía presenta un suave decaimiento con el aumento de la frecuencia y la composición espectral es similar para Iquique y Antofagasta. Arica por su parte, muestra menor energía, consistente con lo observado en los valores diarios. La coherencia entre Iquique y Antofagasta presenta valo-

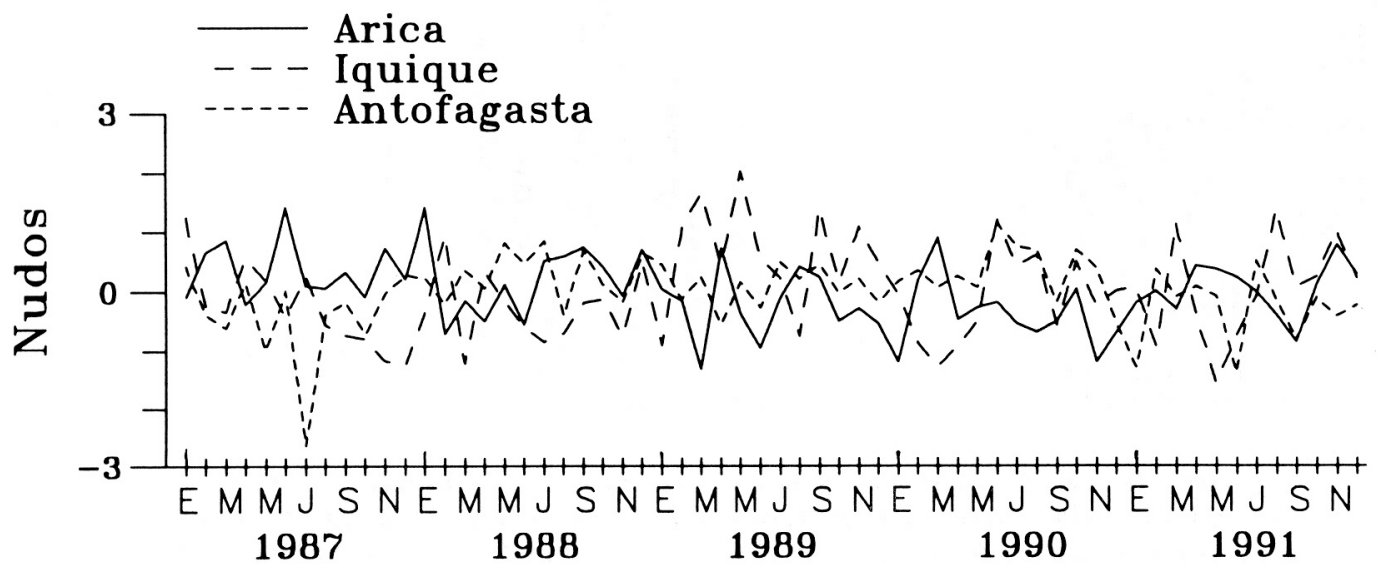

Figura 3. Variación no estacional de la componente paralela a la costa del viento en Arica, Iquique y Antofagasta. Los valores positivos indican viento hacia el norte. 

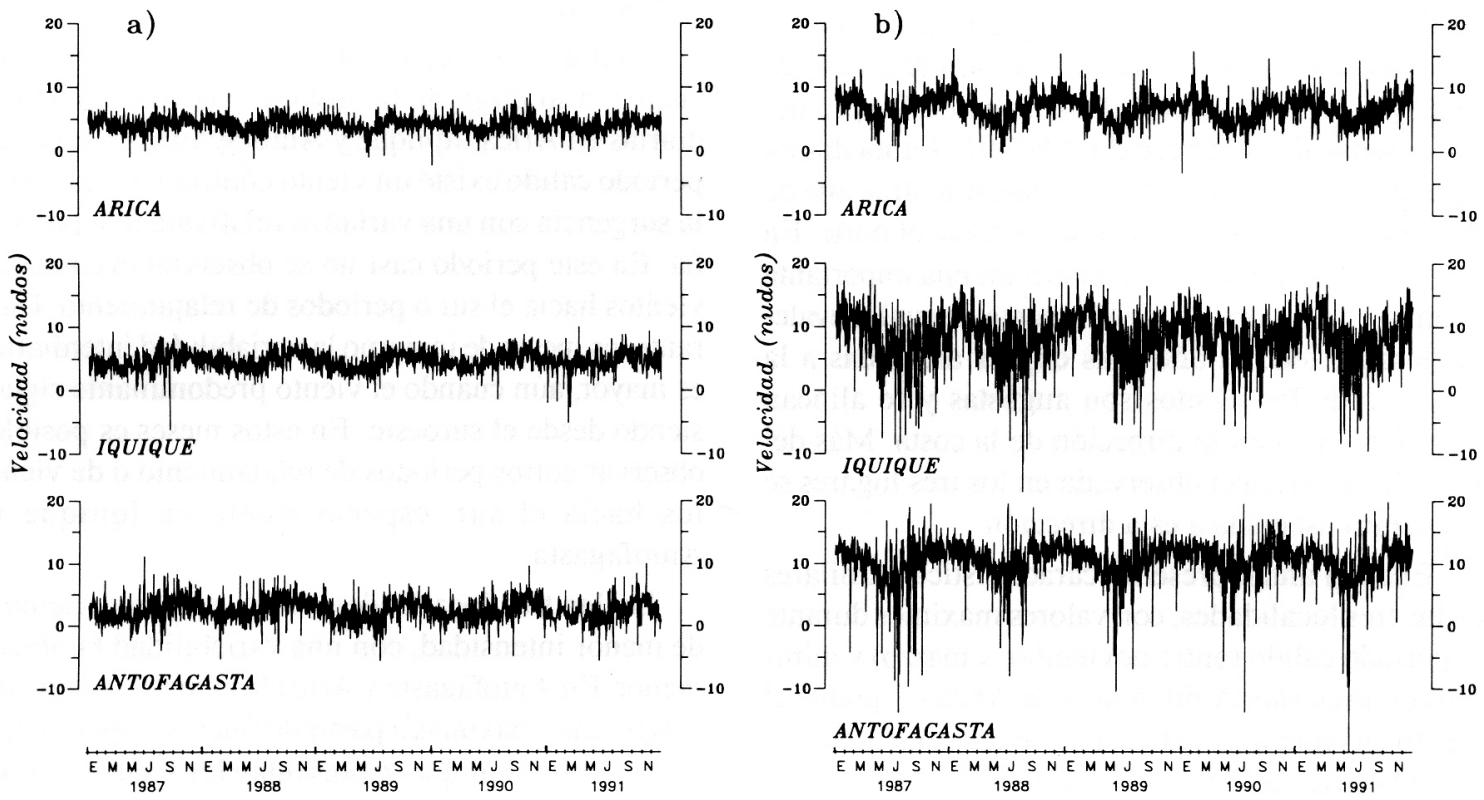

Figura 4. Componentes perpendicular (a) y paralela (b) a la costa de la velocidad del viento en Arica, Iquique y Antofagasta. Los valores positivos en (a) indican que el viento sopla hacia la costa y en (b) aproximadamente hacia el norte.
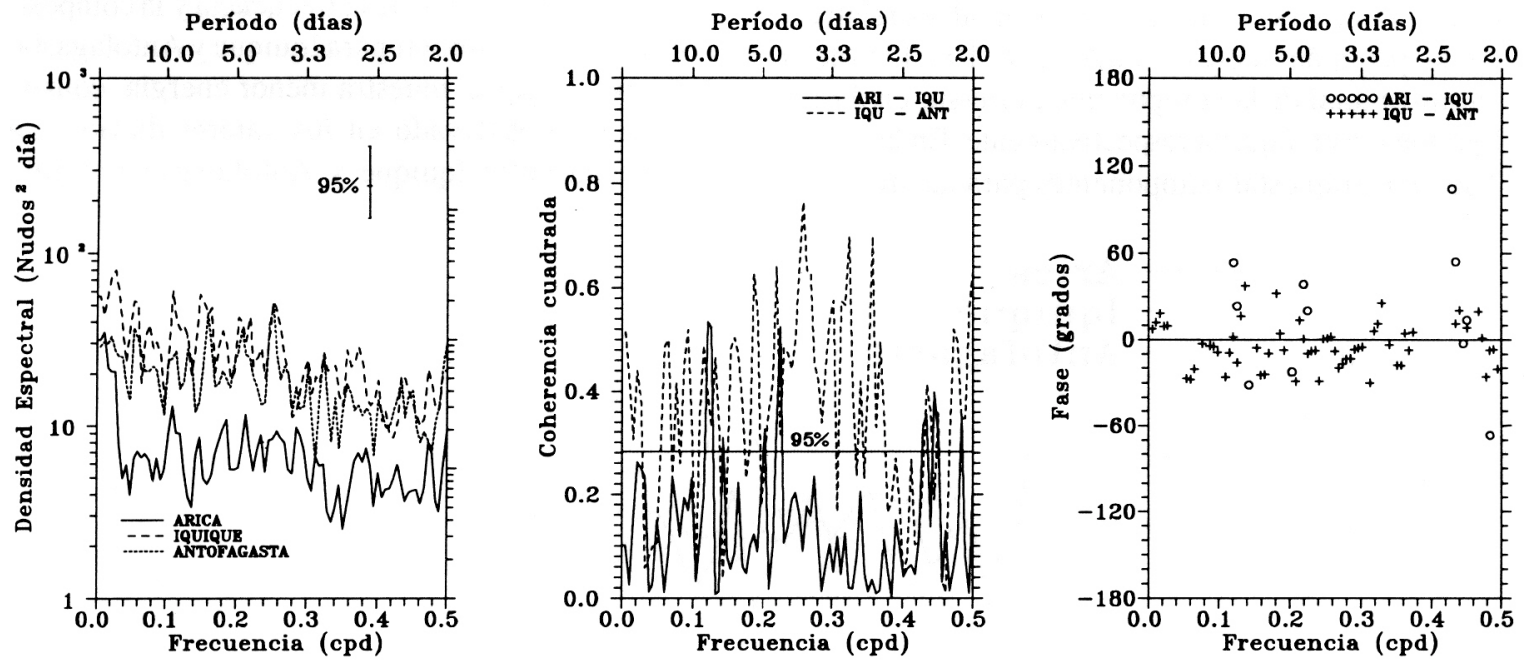

Figura 5. Espectros de energía, coherencia y fase del viento en Arica, Iquique y Antofagasta. Los pares de estaciones para la coherencia se indican al interior de la figura. La línea horizontal en la figura de coherencia indica el límite para las observaciones significativas al 95\% de confianza. La línea vertical en el espectro de energía muestra el intervalo de confianza para las estimaciones. Además se indica la resolución en ancho de banda del espectro. Los valores de la fase fueron graficados sólo para las frecuencias en que la coherencia fue significativa. 
res significativos al 95\% de confianza con máximos centrados entre los 2,5 y 7 días aproximadamente. La fase para estas localidades es variable, pero no supera un rango de \pm 40 grados. Aunque entre lquique y Arica, existe una distancia menor, la coherencia entre las perturbaciones del viento es baja y sólo ocasionalmente alcanza valores significativos. Los espectros de invierno de lquique y Antofagasta (no mostrados aquí) muestan mayor energía que los de verano en toda la banda de frecuencias $(0,25$ meses $^{-1}$ a 0,5 días $\left.^{-1}\right)$, reflejando la mayor variabilidad de la serie durante este período.

\section{El nivel del mar}

En base a los promedios de cada mes, del período 1987-91, se estimó el ciclo anual del NMA en cada localidad. Este no presentó una estacionalidad armónica anual simple y su amplitud, cercana a los 5 $\mathrm{cm}$, fue pequeña en comparación con las variaciones de período menor (Fig. 6). Dado el limitado número de años empleados en el análisis las estimaciones de los ciclos anuales no representa adecuadamente la variación anual caracteristica de la región, especialmente cuando existen fluctuaciones de algunas semanas mucho más energéticas que pueden alterar considerablemente los valores de los

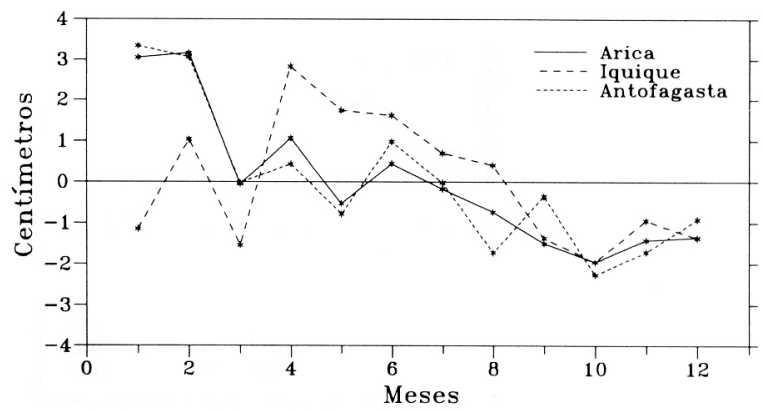

Figura 6. Variación anual promedio del nivel del mar para el período 1987-91, en Arica, Iquique y Antofagasta.

promedios mensuales y cuando, además, existe una importante variación interanual que afecta a la variable. Tal es el caso del período de estudio donde se puede distinguir la fase cálida (año 1987) y fría (1988) de la oscilación del sur.

A escalas de días, el NMA mostró fluctuaciones de varios centímetros de amplitud, las cuales se presentan casi simultáneamente en las tres localidades (Fig. 7). La máxima correlación fue, en general, significativa a un $95 \%$ de confianza. El rasgo más notable

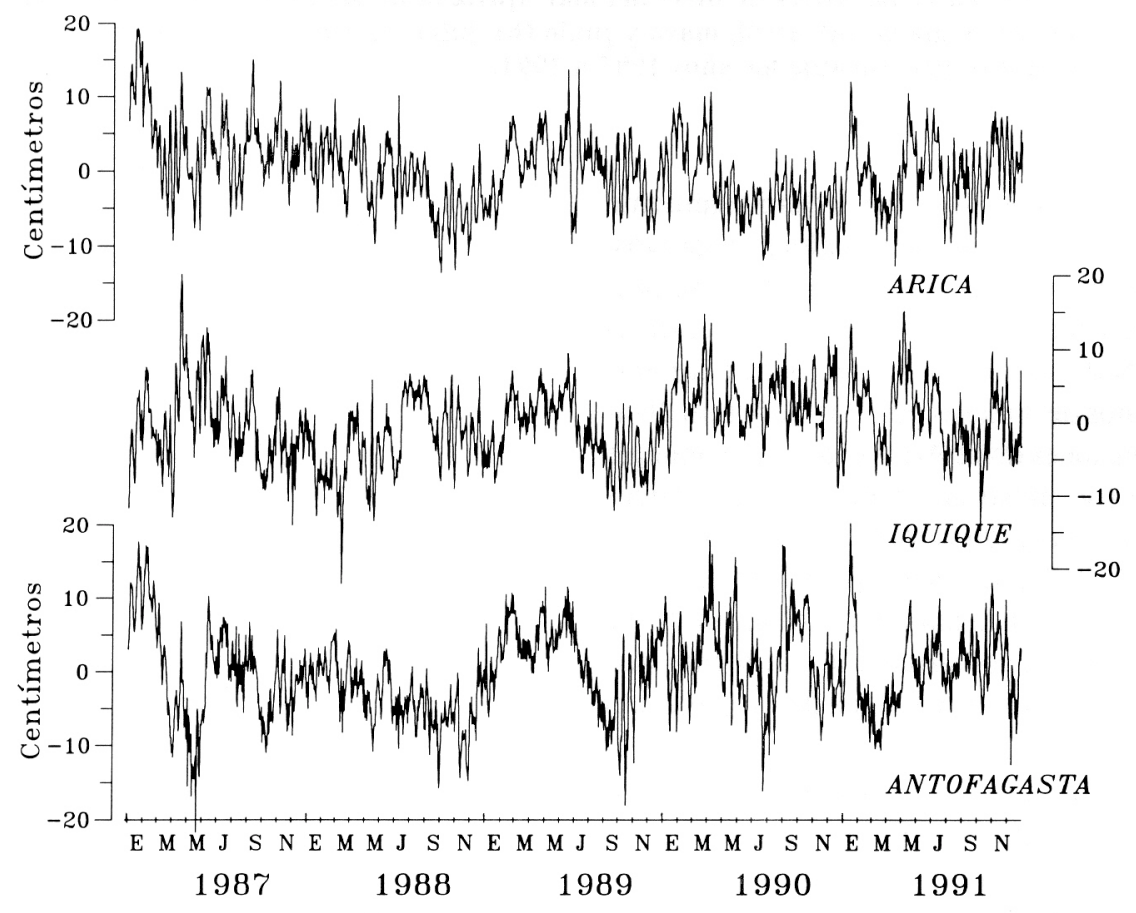

Figura 7. Series de promedios diarios del nivel del mar. 

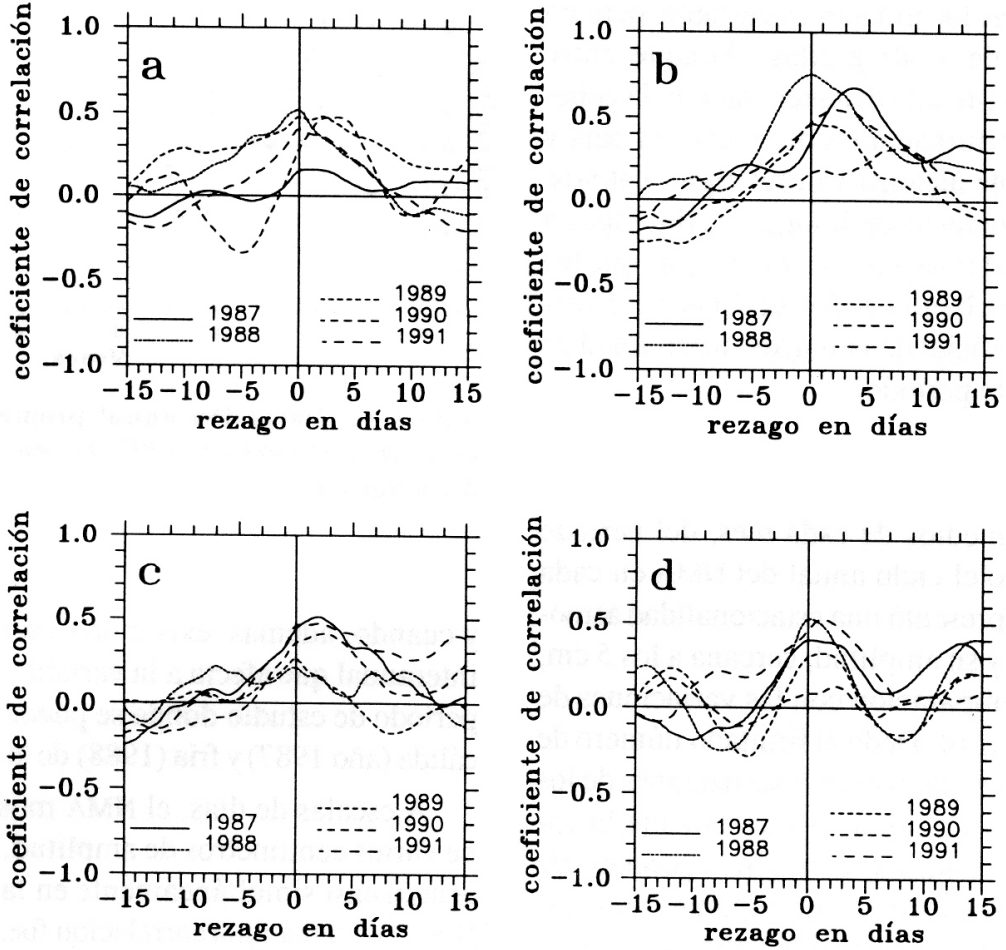

Figura 8. Correlaciones entre las series de nivel del mar ajustado de Arica y Antofagasta, para los meses de enero, febrero y marzo (a), abril, mayo y junio (b), julio, agosto y septiembre (c) y octubre, noviembre y diciembre (d), durante los años 1987 a 1991.

de los correlogramas de NMA fue el desplazamiento de la máxima correlación hacia valores positivos, mostrando que las variaciones en las series se propagan de norte a sur. La Figura 8 muestra la correlación entre el NMA de Arica y Antofagasta separados en segmentos de 3 meses para los 5 años analizados. Las correlaciones entre Arica e Iquique, e Iquique y Antofagasta (no mostradas aquí), tienen tendencias similares alcanzando valores mayores de correlación (entre 0,5 y 0,8 , típicamente para Iquique-Arica) y rezagos menores para la máxima correlación (1 a 2 días en ambos casos), consistente con la menor distancia entre las estaciones y la idea de propagación de norte a sur.

Los espectros de las series de NMA se muestran en la Figura 9, en ellos se observa un importante aumento de energía para períodos mayores a 9 días, con un importante máximo dentro de la banda de 10 a 15 días.

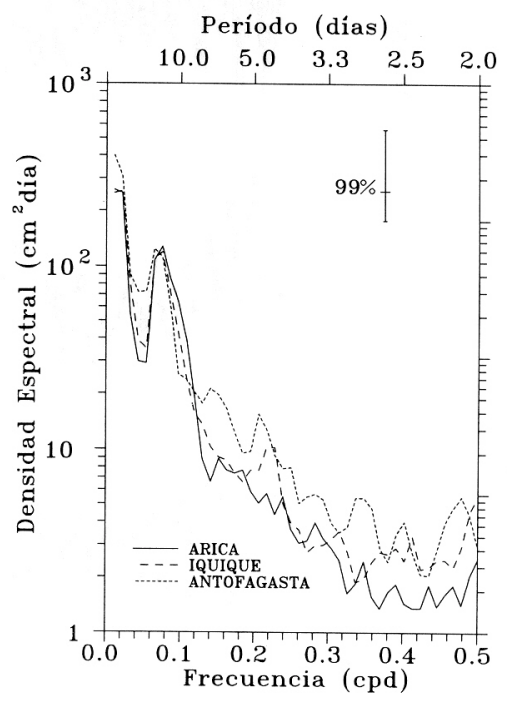

Figura 9. Densidad espectral de las series de nivel del mar ajustado en Arica, Iquique y Antofagasta. 


\section{La temperatura del mar en la costa}

La TMC de Arica y Antofagasta presentó una variación anual predominante, con valores máximos entre $21^{\circ}$ y $23^{\circ} \mathrm{C}$ en verano y mínimos, cercanos a los $15^{\circ} \mathrm{C}$, en invierno. En Iquique la TMC de los meses de verano fue típicamente menor (entre $19^{\circ}$ y $20^{\circ} \mathrm{C}$ ) a la registrada en Arica y Antofagasta, dándole una apariencia menos marcada al ciclo anual (Fig. 10). La TMC de Arica e Iquique exhibió los máximos valores durante el verano de 1987, asociado a la ocurrencia de un evento ENOS. Los valores correspondientes a los meses de invierno de ese año, también fueron mayores a los observados en los otros inviernos. Por otra parte, el verano más frio fue el del año 1989, en este período la TMC de Iquique, no superó los $19^{\circ} \mathrm{C}$.

Cabe destacar que la TMC del verano de 1987 en Antofagasta, no presentó valores mayores a los otros veranos, ni hubo en el verano de 1989 temperaturas más bajas, como en los casos de Iquique y Arica. Sin embargo, el invierno de 1987 muestra temperaturas ligeramente mayores a las registradas en los otros inviernos del período de estudio, evidenciando un posible efecto del ENOS.
Las variaciones con períodos de días no presentaron una correlación significativa entre las diferentes localidades, mientras que a períodos mayores, existió una buena asociación debido al ciclo anual. Los espectros de la temperatura muestran una tendencia similar en las tres localidades, con altos valores en las bajas frecuencias que decaen suavemente a medida que la frecuencia aumenta (Fig. 11).

La coherencia entre las perturbaciones de TMC de Arica e Iquique, muestra valores que son estadísticamente significativos (a un $95 \%$ de confianza) a períodos mayores que 20 días y entre 7 y 10 días. La Figura 11 muestra la fase sólo para las frecuencias en que la coherencia fue significativa al 95\%. Los valores de la fase entre Arica e Iquique, en el rango de frecuencias menores a $0,1 \mathrm{cpd}$, presentan una tendencia a aumentar con el incremento de la frecuencia. Esto puede interpretarse como una evidencia de propagación hacia el Ecuador. Entre Iquique y Antofagasta la coherencia es menor y la fase no muestra una tendencia similar a la señalada anteriormente.

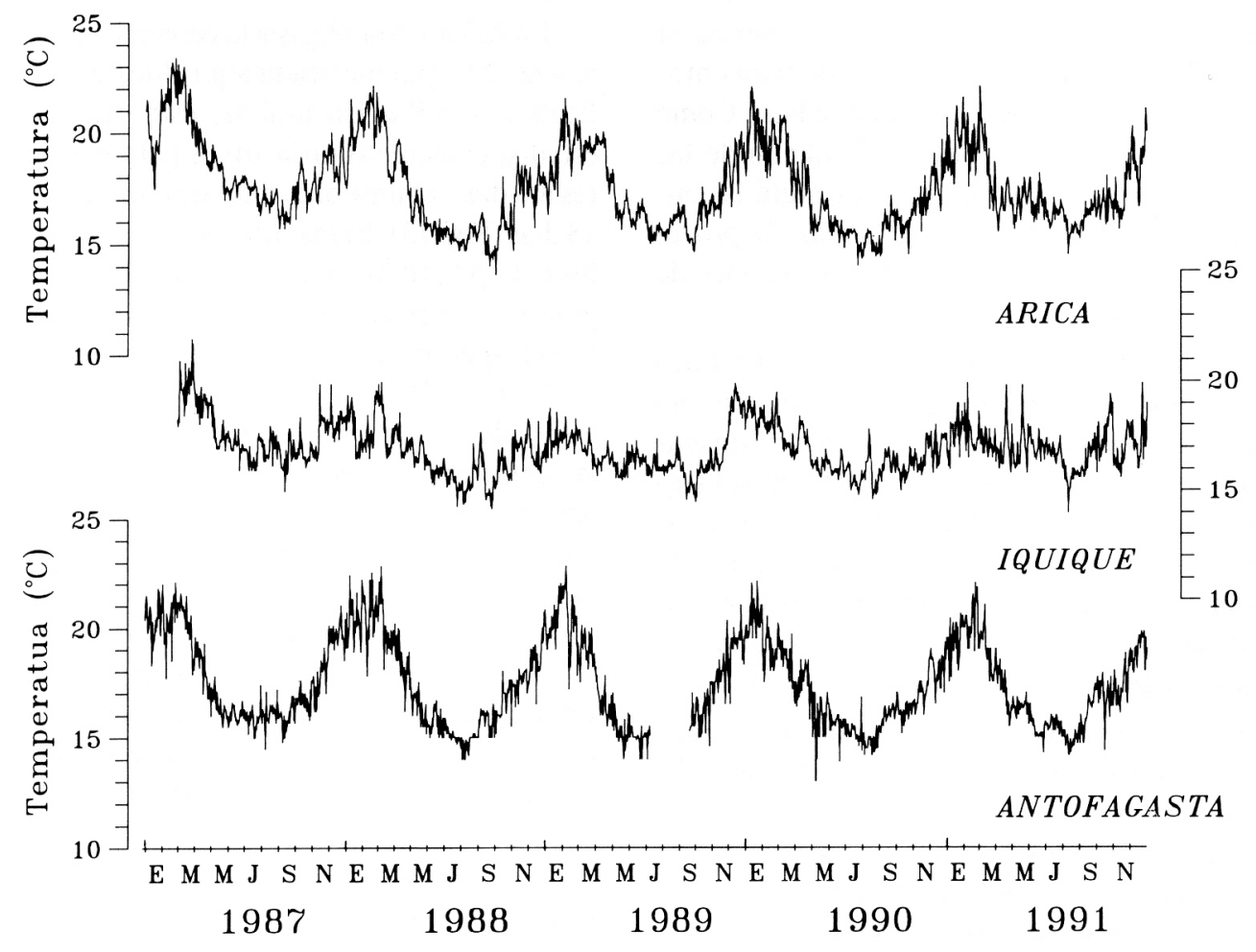

Figura 10. Temperatura del mar en la costa de Arica, Iquique y Antofagasta. Durante el verano las temperaturas de Iquique son inferiores a las de Arica y Antofagasta. 

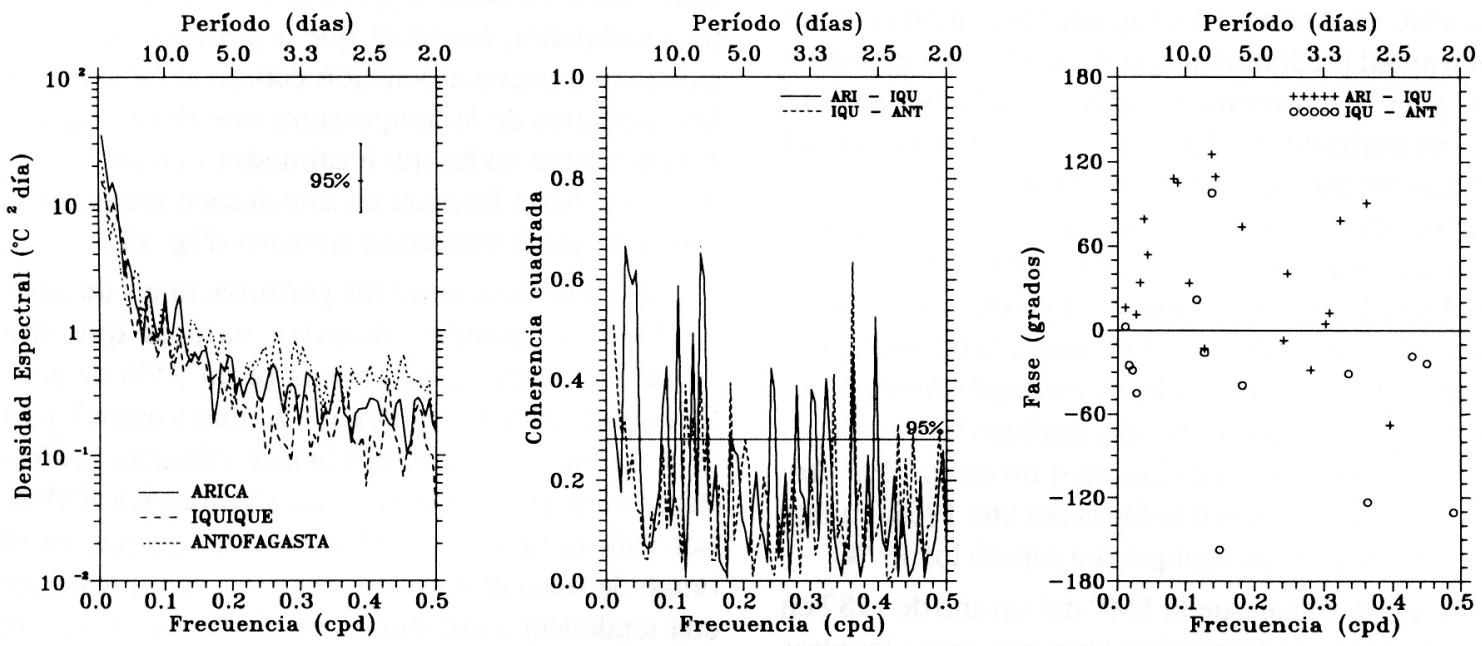

Figura 11. Densidad espectral, coherencia y fase de las series diarias de temperatura de Arica, Iquique y Antofagasta. La fase se muestra sólo para valores de coherencia significativos al $95 \%$.

\section{Perturbaciones originadas por el viento}

La relación entre la componente del viento paralela a la costa y la temperatura del mar a escala interdiaria fue estudiada mediante la correlación de segmentos trimensuales y análisis de coherencia y fase. Como se señaló en la sección 2, previo al cálculo de las correlaciones, a cada segmento se les quitó la tendencia lineal, con el fin de eliminar la relación proveniente de los ciclos anuales y otras variaciones de muy baja frecuencia.

Las máximas correlaciones (en valor absoluto) entre las variables mencionadas anteriormente, no fueron significativas a un $95 \%$ de confianza. Sin embargo, los valores son consistentes en cuanto al signo de la correlación, es decir, para rezagos positivos la correlación es inversa, indicando que un aumento (disminución) del viento implica una disminución (aumento) de la temperatura, en acuerdo con la idea de surgencia de aguas frías forzada por el viento.

Los mayores valores de correlación, en términos absolutos, se registraron en Iquique. En la Figura 12 se muestran estos valores con rezagos entre \pm 7 días para los diferentes años y separadas en períodos de tres meses, enero-marzo, abril-junio, julio-septiembre y octubre-diciembre. Los valores negativos más bajos de correlación se encuentran rezagados entre uno y tres días hacia la derecha, indi- cando que la perturbación en el viento conduce a una perturbación en la temperatura.

En Arica y Antofagasta la coherencia entre el viento y la TMC (no mostrada aquí) fue baja, y no significativa al 95\%. En Iquique ésta presentó valores estadísticamente significativos para períodos mayores a 5 días, con valores más altos en la banda de 6 a 18 días (Fig. 13). El máximo de coherencia, cercano a los $0,1 \mathrm{cpd}$ (10 días), tuvo una fase asociada de 100 grados. Esto puede ser interpretado como un rezago en la respuesta de la temperatura de aproximadamente dos días. Para fluctuaciones de 5 días el rezago estimado fue cercano a un día. Para visualizar mejor las características del rezago entre las perturbaciones del viento y de la TMC, se graftcó la recta relacionada con un desfase igual al período inercial del lugar dentro de la banda de frecuencias entre 0,08 y 0,2 cpd (Fig. 13). En la interpretación de la fase se debe considerar, por hipótesis, que la respuesta es inversa y que las perturbaciones de viento ocurren en primer lugar, es decir, si la temperatura responde en forma simultánea al viento se esperaría una fase de -180 grados.

Para estudiar las relaciones entre el viento y el NMA, se emplearon los análisis de correlación y espectral siguiendo una metodología similar a la empleada para el viento y la TMC. En este caso las co- 

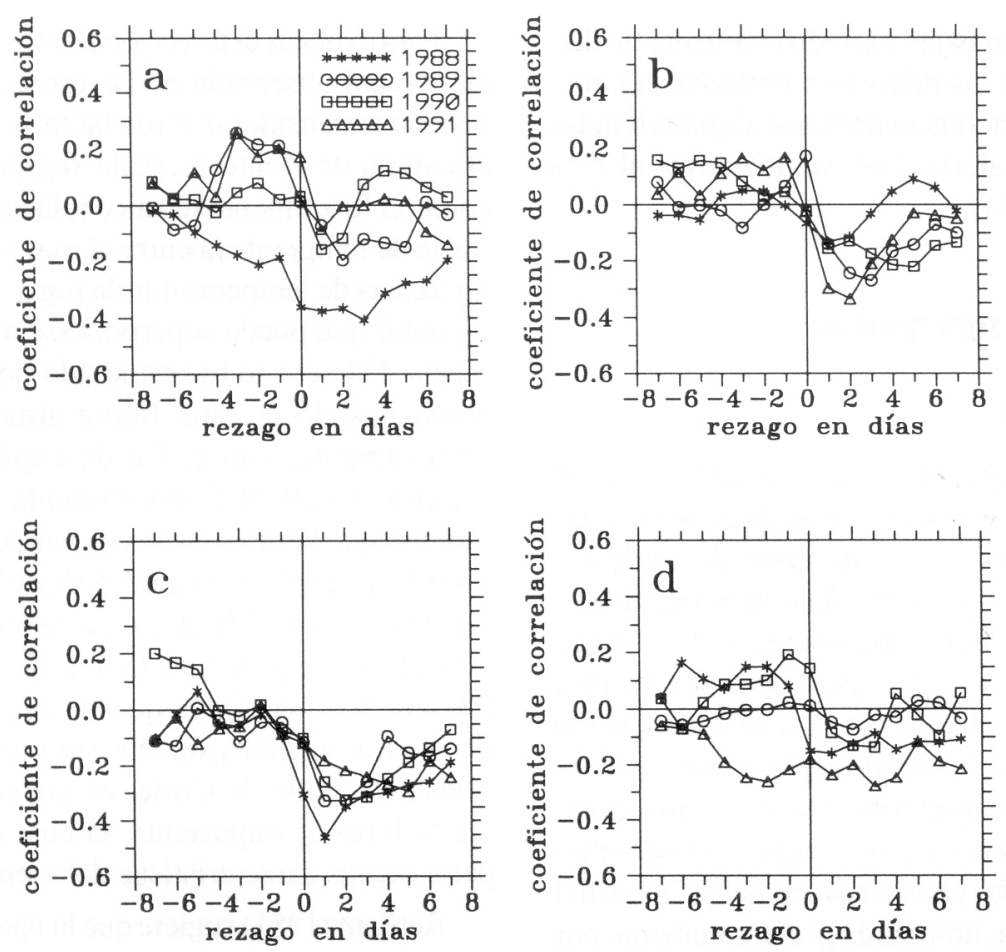

Figura 12. Correlación cruzada entre la componente paralela a la costa del viento y la temperatura del mar en Iquique, separados por año y por períodos trimestrales. (a) enero-marzo, (b) abril-junio, (c) julioseptiembre y (d) octubre-diciembre.
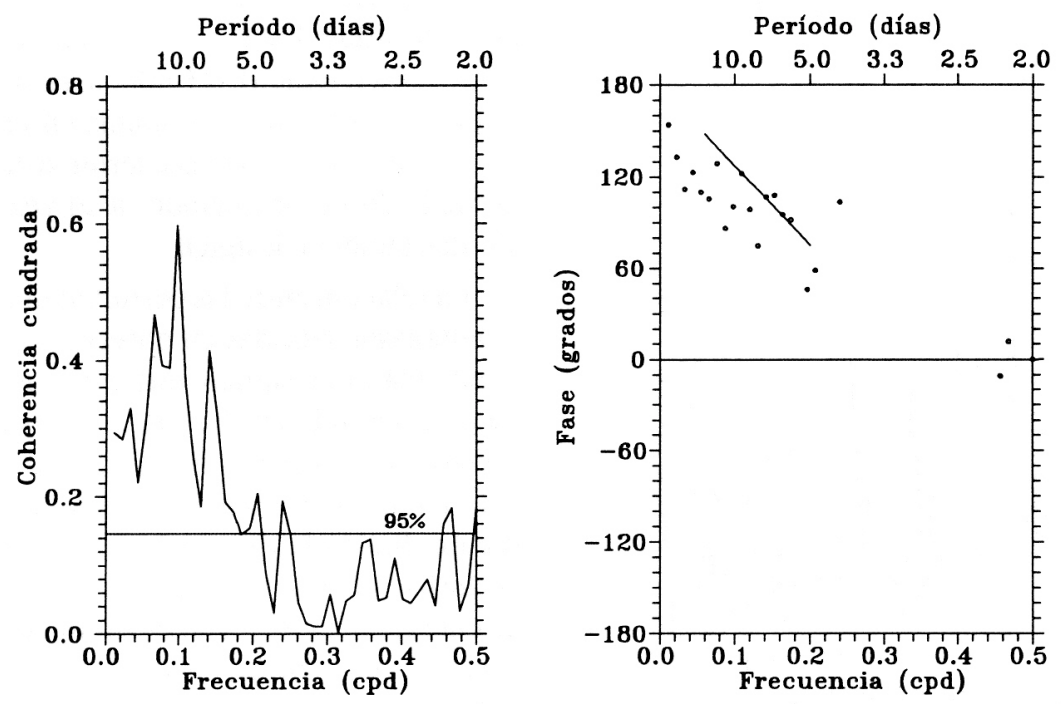

Figura 13. Coherencia y Fase entre la componente del viento a lo largo de la costa y la temperatura del mar en Iquique. La línea en el espectro de fase muestra los valores esperados para un rezago igual al período inercial. La fase se graficó, sólo para valores de coherencia significativos al $95 \%$. 
rrelaciones fueron bajas y estadísticamente no distintas de cero para los diferentes períodos del año. Sin embargo, la coherencia presentó algunas bandas de frecuencia con valores altos, significativos al $95 \%$ de confianza (Fig. 14).

\section{DISCUSION}

\section{Forzamiento local}

Bakun y Nelson (1991) presentaron el ciclo anual del esfuerzo del viento y de su rotor en diferentes zonas de surgencia. Frente a la costa norte de Chile sus resultados muestran un ciclo anual de pequeña amplitud e inverso al observado en la figura 1. Es decir, el esfuerzo del viento en la región oceánica alcanza un máximo durante el invierno. Además, de acuerdo a sus observaciones los vientos promedios bimensuales se encuentran preferentemente en una dirección norte-sur, alineados con la costa. Estos resultados representarían las características del esfuerzo del viento a gran escala, dominada preferentemente por el Anticiclón del Pacífico Sur Oriental.

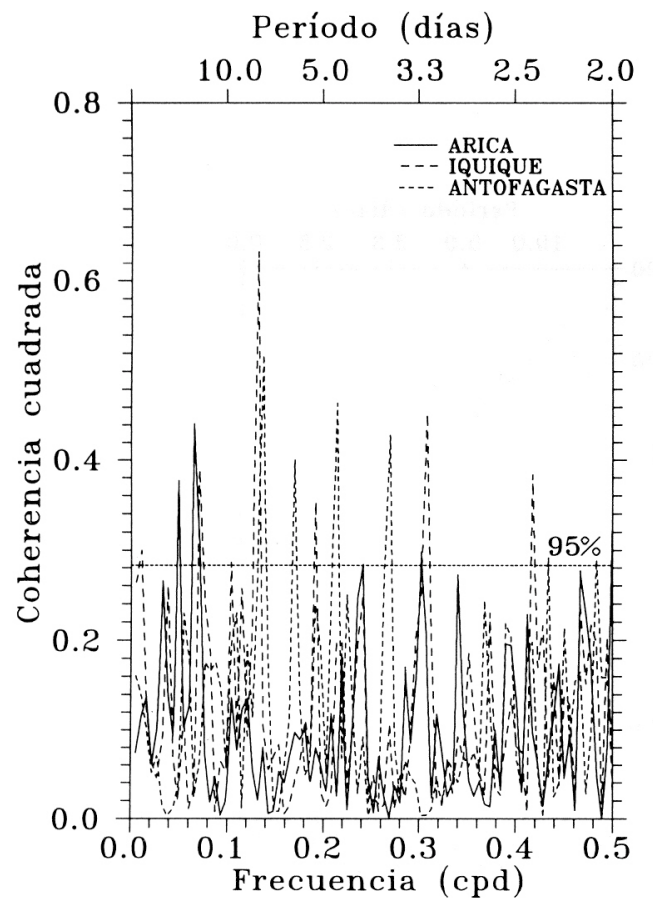

Figura 14. Coherencia entre la componente del viento a lo largo de la costa y el nivel del mar ajustado en Arica, Iquique y Antofagasta.
Para explicar la dirección predominante y la variación anual observada en las estaciones costeras, es necesario considerar otros factores que influyen en el campo de viento a escala regional y local, tales como el forzamiento térmico relacionado con el contraste de temperatura entre el mar y el desierto. Esta diferencia de temperaturas da lugar a un forzamiento regional que puede superponerse al campo de gran escala. Este efecto fue estudiado por Lettau (1978), quien modeló la capa límite atmosférica forzada térmicamente, con el fin de explicar los vientos costeros en el Perú. Posteriormente, Enfield (1981b) muestra que las diferencias de temperatura entre Callao y Lima explican más del $50 \%$ de la varianza de los vientos en Lima. Mientras que la insolación sobre el desierto es la principal fuente de variabilidad del forzante térmico. Dado que las condiciones de temperatura son relativamente similares en las costas áridas del norte de Chile, es razonable esperar un efecto térmico importante, el cual daría cuenta, en parte, de las características del viento costero.

Rutllant (1981) sugiere que la variación estaciona! del viento en la zona de Antofagasta es el resultado de una interacción entre el forzamiento regional (forzamiento térmico) y de gran escala. Los resultados de Montecinos (1991) aportaron evidencias para confirmar este hecho. El forzamiento térmico podría explicar, además, parte de las asimetrías del campo de viento entre las tres localidades y el desfase en el ciclo anual observado en Iquique. Ya que los efectos de nubosidad local y la presencia de zonas de surgencias más activas (inducidas, por ejemplo, por la presencia de cabos o puntas en la línea costera) darían lugar a un contraste térmico desigual a lo largo de la costa; originando, a su vez, un campo de vientos también desigual.

La relación entre las perturbaciones de Iquique y Antofagasta, basadas en observaciones en horas de viento máximo, sugiere que existe una componente en la variabilidad de los vientos superficiales que es forzada por la escala sinóptica. Durante el invierno los vientos presentan una disminución en toda la región. Sin embargo, en Iquique y Antofagasta las perturbaciones muestran un importante incremento de energía (acompañado de un ligero aumento de la coherencia, no mostrada aquí). La disminución de la radiación solar en este período debilita la componente del viento forzada térmicamente (Montecinos, 1991). Es probable que el incremento de las perturbaciones invernales esté asociado a un incremento de las 
fluctuaciones de gran escala. Adicionalmente, la presencia de perturbaciones de escala regionallocal como bajas costeras, pueden jugar un papel significativo en las fluctuaciones observadas.

Otro aspecto que debe tenerse en cuenta en el análisis del viento, es la ubicación de las estaciones donde se efectúan las mediciones. En este caso, las estaciones meteorológicas están localizadas al interior de aeropuertos las cuales pueden ser influenciadas por la topografia del lugar (Montecinos, 1991). La presencia de los cerros costeros que conforman la cordillera de la costa constituyen una importante restricción a la dirección del viento. En el caso de Antofagasta la dirección de los vientos de la tarde medidos en el aeropuerto Cerro Moreno son casi exclusivamente del sur durante todo el año, contrastando con observaciones efectuadas en puntos relativamente cercanos tales como la Universidad Católica del Norte, donde existe un predominio de vientos provenientes del sudoeste (Zuleta, 1988). Estas observaciones dejan de manifiesto la influencia del factor topográfico que modifica la dirección del campo de viento en las cercanías del aeropuerto. Posiblemente, la presencia de grandes cerros a ambos lados (este y oeste) canalizan el viento en una dirección sur-norte. Los efectos topográfico s sobre el viento en las tres estaciones fue discutido en mayor detalle por Montecinos (1991).

En algunos casos los efectos topográficos pueden tener un pequeño alcance espacial, limitando la representatividad de la información para el estudio de fenómenos de mayor escala. Mientras que rasgos topográficos mayores pueden tener una influencia importante en las características del viento a una escala mayor, influyendo directamente en los fenómenos oceanográficos. Tal parece ser el caso del cambio de dirección de la línea de costa al norte de Arica. Los vientos sobre el océano frente a esta región presentan un notable cambio de dirección y típicamente las magnitudes son menores (Bakun y Nelson, 1992).

Una característica importante de destacar, es la diferencia entre los espectros de viento de la región y los espectros de latitudes mayores. En la zona norte de Chile el viento no presenta un aumento de energía en la banda de actividad ciclónica (2 a 7 días). De tal modo que, durante el verano, cuando los vientos favorables a la surgencia son máximos, no se observan períodos de relajamiento o de vientos en sentido contrario, como ocurre en las zonas de surgencia de Chile central o en
Oregon y norte de California en el Pacífico Nororiental (Huyer, 1983). Esta variabilidad genera pulsos de surgencia que originan, a su vez, una distribución de propiedades (temperatura, salinidad, nutrientes, etc.) que es muy heterogénea espacial y temporalmente. En la zona norte de Chile, durante el período de vientos más intensos se esperaría una surgencia más estable; sin embargo, las oscilaciones relativamente pequeñas pueden producir perturbaciones significativas ya que éstas actúan sobre un sistema cuya condición media es de surgencia (la cual se podría considerar como estacionaria dentro de una escala de tiempo de algunos días).

Durante los meses de verano los vientos hacia el norte son más intensos y persistentes en el tiempo, lo cual permite suponer que la surgencia es más activa. Sin embargo, el ciclo anual de la TMC y de la componente paralela a la costa del viento, se encuentran en fase, con máximos en verano y mínimos en invierno. Esto muestra que la variación anual de la TMC no es dominada por el ascenso de aguas frías durante el verano asociado a la surgencia costera.

De las tres localidades estudiadas, tanto la correlación cruzada como el análisis espectral mostraron que en Iquique existe una relación inversa entre perturbaciones del orden de días de la TMC y la componente del viento a lo largo de la costa, ajustándose a lo esperado en una región de surgencia costera. La alta coherencia entre 0,06 y 0,12 cpd (16.7 y 8.3 días) indica la escala temporal en que mejor se relaciona el forzante (viento a lo largo de la costa) y la respuesta (disminución de la temperatura en la costa asociada a eventos de surgencia). Por su parte, la fase indica que, en este rango de frecuencias, el rezago en la respuesta es entre uno y dos días.

Las evidencias encontradas en la zona de Iquique permiten suponer que la anomalía del ciclo anual en la temperatura del mar de Iquique, en relación a los ciclos de Arica y Antofagasta, son producto de la intensificación de los vientos durante el verano. Es posible que esta relación no se presente en Arica y Antofagasta por la menor intensidad de la surgencia en esas localidades o, simplemente, los registros costeros de temperatura o de viento no representan adecuadamente el régimen sobre el margen continental. La solución a esta disyuntiva requiere de mediciones del campo de viento sobre el océano y de una validación de la temperatura de los mareógrafos en base a registros cercanos a la costa, pero alejados del borde litoral. Por otra parte, la relación vientoTMC encontrada en Iquique a escalas de días mues- 
tra que estos registros son adecuados, al menos en una primera aproximación, para representar los fenómenos oceanográficos costeros.

De acuerdo al esquema clásico de surgencia costera se debería esperar una relación inversa entre la componente del viento paralela a la costa y el NMA En este trabajo la coherencia entre ambos registros mostró gran variabilidad a diferentes frecuencias, destacándose algunos picos de valores altos en bandas específicas. El valor observado entre 0,12 y 0,14 cpd es estadísticamente signíficativa a un $99 \%$ de confianza y constituye una evidencia de forzamiento local producido por el viento. Posiblemente la gran variabilidad del nivel del mar originada remotamente encubre, en cierta medida, su relación con el viento. Pizarro (1991) analizó la relación entre viento y NMA en diferentes puntos de la costa de Chile empleando información del año 1986. En Antofagasta el autor encontró que las perturbaciones de ambas variables se relacionaron significativamente dentro de una banda centrada en los 10 días de período. El desfase entre las variables en esta banda, fue cercano a los $180^{\circ}$, reflejando una relación inversa, con un tiempo de respuesta cercano a un día.

\section{Ondas atrapadas a la costa}

Diversos trabajos han mostrado la existencia de perturbaciones del nivel del mar que se propagan como ondas atrapadas en la costa oeste de Sudamérica (Smith, 1978, Romea y Smith, 1983, Enfield y Allen, 1980, Enfield, 1987, Enfield, et al., 1987, CornejoRodríguez y Enfield, 1987). Actualmente se ha logrado demostrar que gran parte de estas perturbaciones tienen un origen ecuatorial y son el resultado de la intensa interacción entre el océano y la atmósfera en bajas latitudes. Las perturbaciones en los vientos zonales sobre el Pacífico Ecuatorial Central generan ondas que viajan hacia la costa de Sudamérica y una vez que ellas interactúan con el borde continental pueden transformarse en ondas atrapadas a la costa irradiando parte de su energía hacia los polos. Estas oscilaciones, aunque tienen una expresión de sólo algunos centímetros en el nivel del mar, perturban la picnoclina varias decenas de metros, afectando la estructura térmica y distribución de masa en las regiones costeras modificando, además, las características locales de la respuesta del océano al viento.

Los rezagos de las máximas correlaciones (Fig. 8) muestran que las variaciones del NMA viajan de norte a sur con una velocidad tal que involucra un desfase entre 1 y 3 días, lo cual implica una velocidad de propagación entre 200 y $600 \mathrm{~km} /$ día aproximadamente. Sin embargo, el desfase obtenido del análisis de correlación no permite resolver adecuadamente esta velocidad ni las frecuencias de las oscilaciones involucradas. En este sentido, el análisis espectral es una herramienta más adecuada.

La Figura 15 muestra los espectros de coherencia y fase entre el NMA de Arica e Iquique. Si las perturbaciones son ondas no dispersivas, es decir, que viajan con igual velocidad independientemente de su período, la variación del ángulo con la frecuencia en el espectro de fase, debería ser una recta que pasa por el origen, cuya pendiente depende de la velocidad con que viajan las perturbaciones. Basándose en esta suposición se ajustó una recta a los valores de la fase con frecuencias menores a 0,2 ciclos por día (Fig. 15). De acuerdo a esta estimación, la velocidad de propagación fue de $300 \mathrm{~cm} / \mathrm{s}$ (260 km/día). Este valor se ajusta a los observados en Perú (Smith, 1978; Romea y Smith, 1983 y Cornejo-Rodríguez y Enfield, 1987) y a los obtenidos por Brink (1982) para ondas híbridas, no forzadas, atrapadas a la costa. Valores similares fueron encontrados por Pizarro (1991) en la misma zona de estudio.

Los trabajos efectuados en las costas de Perú (Smith, 1978, Enfield y Allen, 1980, Romea y Smith, 1983; Enfield, 1987, Enfield, et al., 1987, CornejoRodríguez y Enfield, 1987) permiten suponer que gran parte de la variabilidad presente en el nivel del mar de la zona norte de Chile, tiene un origen remoto. Esto, al igual que en las costas de Perú, encubriría una posible relación existente entre el viento local y el nivel del mar. De hecho, la presencia de ondas atrapadas a la costa dominan la correlación y un amplio rango en el espectro. Las ondas, inicialmente baroclínicas (tipo ondas Kelvin internas), son capaces de transformarse en ondas híbridas (afectadas por la topografía y la estratificación) a medida que se propagan hacia los polos y disminuye el radio interno de deformación. De esta manera, la energía proveniente de la zona ecuatorial, es capaz de propagarse en forma eficiente hacia latitudes mayores (Allen y Romea,1980).

\section{CONCLUSIONES}

A lo largo de la costa norte de Chile predomina el viento proveniente del sudoeste durante todo el año. 

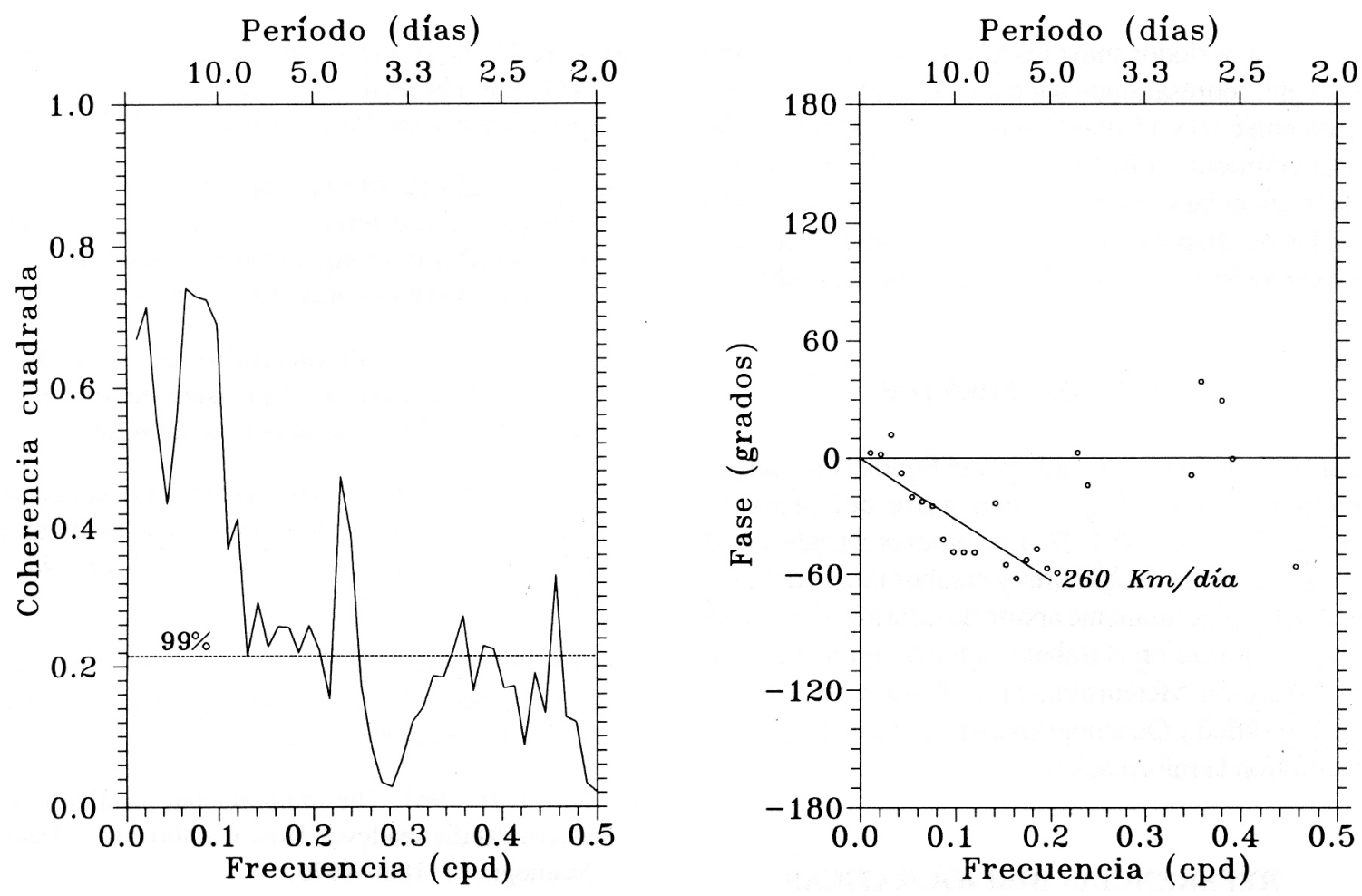

Figura 15. Coherencia y fase entre las series diarias de nivel del mar de Arica e Iquique. La fase se graficó sólo para los valores de coherencia significativos al 99\%. A los puntos ubicados bajo los 0,2 ciclos por día se les ajustó una recta por mínimos cuadrados, dejando fijo el origen. Esta muestra que las perturbaciones del nivel del mar viajan como ondas no dispersivas a una velocidad de 260 km/día.

Este presenta una variación anual caracterizada por valores máximos en verano y mínimos en invierno. El eje principal sobre el cual ocurren las perturbaciones se orienta en una dirección aproximadamente norte-sur paralelo a la costa, a diferencia de la dirección media. Las perturbaciones del viento presentan una variación anual, con máximas amplitudes en invierno y mínimas en verano. El viento predominante tiene una componente que es favorable a la surgencia, la cual prevalece durante casi todo el año. Esta es más intensa y estable en verano. En invierno, el viento favorable a la surgencia se presenta en forma de pulsos o eventos, alternándose con periodos de calma o viento desde el norte.

La temperatura del mar en la costa tiene un importante ciclo anual, el cual se encuentra en fase con el viento. De modo que la surgencia no juega un rol dominante en la definición de este ciclo. Sin embargo, el enfriamiento costero debido a este fenómeno produce importantes anomalías en algunos puntos de la costa. Los datos de TMC de Arica y Antofagasta no muestran buena relación con el viento, mientras que en Iquique ambas variables se relacionan en forma inversa a escalas de días. Esto explicaría las anomalías negativas de la TMC observadas durante el verano en Iquique, en relación a los valores de Arica y Antofagasta. La mejor relación entre las perturbaciones de viento y TMC en Iquique se presentó para variaciones entre 6 y 18 días con un rezago estimado entre 1 y 2 días.

En general, el nivel del mar no se relaciona bien con el viento, aunque dentro de ciertas bandas de frecuencia se observaron valores significativos. En Iquique y Antofagasta destaca la banda centrada a los 0,13 cpd (7,7 días) con valores de coherencia significativos a un $99 \%$ de confianza. Esta relación evidenciaría la existencia de un forzamiento local sobre las variaciones del nivel del mar. Sin embargo, al igual que en las costas del Perú, las ondas atrapadas a la costa dominan la variabilidad de baja frecuencia del nivel del mar y sólo una pequeña fracción de esta última podría ser atribuída a un forzamiento atmosférico local.

Las variaciones de baja frecuencia del nivel del 
mar, con períodos mayores a 40 días, presentan alta energía, sobresaliendo además, una banda comprendida entre 10 y 15 días. Estas perturbaciones fueron espacialmente coherentes y en general, en un rango de frecuencias menores a $0,2 \mathrm{cpd}$, se comportan como ondas no dispersivas que viajan de norte a sur con una velocidad estimada de $300 \mathrm{~cm} / \mathrm{s}$ ( $260 \mathrm{~km} /$ día $)$.

\section{AGRADECIMIENTOS}

Este trabajo fue financiado por el Fondo Nacional de Ciencia y Tecnología como parte del proyecto FONDECYT N ${ }^{\mathrm{O}} 0481 / 92$. Los autores agradecen al conjunto de investigadores y colaboradores del proyecto por el permanente aporte de información e ideas que enriquecieron el trabajo. Además agradecemos a la Dirección Meteorológica de Chile y al Servicio Hidrográfico y Oceanográfico de la Armada quienes facilitaron la información.

\section{REFERENCIAS BIBLIOGRAFICAS}

ALLEN, J.S., and R.D. ROMEA. 1980. On coastal trapped waves at low latitudes in stratified ocean. J. Fluid. Mech., 93: 555-585.

ARCOS, D.F. y N. NAVARRO. 1986. Análisis de un índice de surgencia para la zona de Talcahuano. Chile (latitud $37^{\circ} \mathrm{S}$ ). Invest. Pesq. Chile, 33: 91-98.

BAKUN, A. and C. NELSON. 1991. The seasonal cycle of wind stress curl in subtropical eastern boundary current regions. J Phys. Oceanogr., 21: $1815-1834$

BEARDSLEY, R., C. DORMAN, A. FRIECHE, K. ROSENFELD and C. WINNANT. 1987. Local atmospheric forcing during the Coastal Ocean Dynamical Experiment. 1. A description of the marine boundary layer and atmospheric conditions over a northern California upwelling region. J. Geophys. Res., 92(C2): 1647-1488.

BENDAT, J.S. and A.G. PIERSOL. 1972. Random data: Analysis and measurement procedures. WileyInterscience, $407 \mathrm{pp}$.

BRINK, K.H. 1982. A comparison of long coastal trapped wave theory with observations off Peru J. Phys. Oceanogr., 12: 897-913.

BRINK, K.H., D. HALPERN, A. HAYER and R. SMITH. 1983. The physical environment of the peruvian upwelling system. Prog. Oceanogr., 12: 285-305.

CORNEJO-RODRÍGUEZ, M. DEL P. and D.B. ENFIELD. 1987. Propagation and forcing of high-frequency sea level variability along the west coast of South America. J. Geophys. Res., 92(C13): 14323-14334.

DAVIES, R.E. 1976. Predictability of sea surface temperature and sea level pressure anomalies of the Pacifico J. Phys. Oceanogr., 6: 249-266.

ENFIELD, D.B. 1981a. Annual and no seasonal variability of monthly low level wind fields over the Southeastern Tropical Pacifico Mon. Wea. Rev., 109: 2177-2190.

ENFIELD, D.B. 1981b. Thermally driven wind variability in the planetary boundary layer above Lima, Peru. J. Geophys. Res., 86(C3): 2005-2016.

ENFIELD, D.B. 1987. The intraseasonal oscillation in eastern Pacific sea level: How is it forced?. J. Phys. Oceanogr., 17: 1860-1876.

ENFIELD, D.B. and J.S. ALLEN. 1980. On the structure and dynamics of monthly sea level anomalies along the Pacific coast of North and South America. J. Phys. Oceanogr., 10: 557-578.

ENFIELD, D.B., M. DEL P. CORNEJORODRÍGUEZ, R.L. SMITH and P.A. NEWBERGER. 1987. The ecuatorial source of propagating variability along the Perú coast during the 1882-1983, J. Geophys. Res., 92(C13): 1433514346.

FONSECA, T. 1987. Anomalías de temperatura y nivel del mar en la costa del pacífico sudoriental. Cienc. Tec. del Mar, CONA, 11: 17-43.

FUENZALIDA, H. 1971., Climatología de Chile, Publicación interna, Departamento de Geofísica, Univ. de Chile, 73 pp.

FUENZALIDA, R. 1990. Variabilidad temporal de un índice de surgencia para la zona de Iquique (latitud $20^{\circ} \mathrm{S}$ ). Invest. Cient. y Tec., Serie: Ciencias del Mar, Iquique, 1: 37-47.

HUYER, A. 1983. Coastal upwelling in the California Current System. Prog. Oceanogr., 12: 259-284.

HUYER, A., R.L. SMITH and T. PALUSZKIEWICZ. 1987. Coastal upwelling off Peru during normal and El Niño times, 1981-1984. J. Geophys. Res., 92(C13): 14297-14307. 
HUYER, A., M. KNOLL, T. PALUSZKIEWICZ and R.L. SMITH. 1991. The Peru Undercurrent: a study in variability. Deep-Sea Res., 38, Supl. 1: S247-S271.

LETTAU, H.H. 1978. Explaining the world's driest climate, in Exploring the World's Driest Climate, editado por H.H. Lettau y K. Lettau IES Rep. 101, Univ. of Wisconsin, Madison.

McCREARY, J.P. 1976. Eastern tropical ocean response to changing wind systems with application to El Niño. J. Phys. Oceanogr., 6: 632-645.

MONTECINOS, A. 1991. Efecto del fenómeno El Niño en los vientos favorables a la surgencia costera en la costa norte de Chile. Tesis de Oceanógrafo, Escuela de Ciencias del Mar, Univ. Católica de Valparaíso, 143 pp.

PIZARRO, O. 1991. Propagación de fluctuaciones de baja frecuencia del nivel del mar a lo largo de la costa de Chile, Tesis de Oceanógrafo, Escuela de Ciencias del Mar, Univ. Católica de Valparaíso, 110 pp.

Recibido el 31 de marzo de 1994.

Aceptado el 4 de noviembre de 1994.
ROMEA, R.D. and R.L. SMITH. 1983. Further evidence for coastal trapped waves along the Peru coast, J. Phys. Oceanogr., 13: 1341-1356.

ROMERO, H. y A.M. GARRIDO. 1985. Influencias genéticas del fenómeno El Niño sobre los patrones climáticos de Chile. Invest. Pesq. Chile, 32: 19-35.

RUTLLANT, J. 1981. Variabilidad estacional e interanual de la velocidad media del viento en Antofagasta y su relación con la temperatura superficial del mar y otros parámetros. Trabajo presentado en las I Jornadas de Ciencias del Mar, $15 \mathrm{pp}$.

SCIREMAMANO, F. 1979. A suggestion for the presentation of correlation and their significance levels. J. Phys. Oceanogr., 9: 1273-1276.

SMITH, R.L. 1978. Poleward propagating perturbations in currents and sea level along the Peru coast. J. Geophys. Res., 83: 6083-6092.

ZULETA, R. 1988. Boletín de información climatológica en Antofagasta. Universidad del Norte, Departamento de Ciencias Físicas, 49 pp. 